PROCEEDINGS OF THE

AMERICAN MATHEMATICAL SOCIETY

Volume 134, Number 8, Pages 2373-2382

S 0002-9939(06)08228-1

Article electronically published on February 8, 2006

\title{
TRACES AND SOBOLEV EXTENSION DOMAINS
}

\author{
PETTERI HARJULEHTO
}

(Communicated by David Preiss)

\begin{abstract}
Assume that $\Omega \subset \mathbb{R}^{n}$ is a bounded domain and its boundary $\partial \Omega$ is $m$-regular, $n-1 \leq m<n$. We show that if there exists a bounded trace operator $T: W^{1, p}(\Omega) \rightarrow B_{1-\alpha}^{p}(\partial \Omega), 1<p<\infty$ and $\alpha=\frac{n-m}{p}$, and $(1-\lambda)$ Hölder continuous functions are dense in $W^{1, p}(\Omega), 0 \leq \lambda<n-m$, then the domain $\Omega$ is a $W^{1, p}$-extension domain.
\end{abstract}

\section{INTRODUCTION}

We say that a domain $\Omega \subset \mathbb{R}^{n}$ is a $W^{1, p}$-extension domain if there exists a bounded linear extension operator $E: W^{1, p}(\Omega) \rightarrow W^{1, p}\left(\mathbb{R}^{n}\right)$, so that $\left.E u\right|_{\Omega}=u$ for every $u \in W^{1, p}(\Omega)$. From a philosophical point of view, when we extend a function it does not matter how irregular the function is inside the domain as long as it is smooth enough near the boundary. Thus it is natural to try to characterize the extension property by traces.

A. Jonsson showed that a trace of a Sobolev function $u \in W^{1, p}\left(\mathbb{R}^{n}\right)$ in an $m$ regular $F \subset \mathbb{R}^{n}, 0<m<n$, belongs to a Besov space $B_{1-\frac{n-m}{p}}^{p}(F)$, 4, Theorem 3, p. 4]. A bounded set $F \subset \mathbb{R}^{n}$ is $m$-regular if

$$
C^{-1} r^{m} \leq H^{m}\left(B^{n}(x, r) \cap F\right) \leq C r^{m}
$$

for every $x \in F$ and every $r, 0<r \leq \operatorname{diam}(F)$. There also exists a bounded extension operator $B_{1-\frac{n-m}{p}}^{p}(F) \rightarrow W^{1, p}\left(\mathbb{R}^{n}\right)$, [4, Theorem 3, p. 4]. However, the problem is that the extension of $u \in W^{1, p}(\Omega)$ should have weak derivatives across the boundary.

Let $1<p<\infty$. Assume that $\Omega \subset \mathbb{R}^{n}$ is a bounded domain and its boundary $\partial \Omega$ is $m$-regular, $n-1 \leq m<n$. Assume that $(1-\lambda)$-Hölder continuous functions are dense in $W^{1, p}(\Omega), 0 \leq \lambda<n-m$. Assume that there exists a bounded linear trace operator

$$
T: W^{1, p}(\Omega) \rightarrow B_{1-\frac{n-m}{p}}^{p}(\partial \Omega),
$$

so that $T u(x)=\lim _{r \rightarrow 0} f_{B^{n}(x, r) \cap \Omega} u(z) d H^{n}(z) H^{m}$-almost everywhere in $\partial \Omega$. Then we show that the domain $\Omega$ is a $W^{1, p}$-extension domain.

Received by the editors October 26, 2000 and, in revised form, March 10, 2005

2000 Mathematics Subject Classification. Primary 46E35.

Key words and phrases. Sobolev space, Besov space, trace operator, extension operator.

(C)2006 American Mathematical Society Reverts to public domain 28 years from publication 
Jonsson's theorem implies the following result. Assume that $\Omega \subset \mathbb{R}^{n}$ is a bounded $W^{1, p}$-extension domain and its boundary $\partial \Omega$ is $m$-regular, $n-1 \leq m<n$. Then there exists a bounded trace operator

$$
T: W^{1, p}(\Omega) \rightarrow B_{1-\frac{n-m}{p}}^{p}(\partial \Omega)
$$

so that $T u(x)=\lim _{r \rightarrow 0} f_{B^{n}(x, r) \cap \Omega} u(z) d H^{n}(z) H^{m}$-almost everywhere in $\partial \Omega$.

We obtain a characterization for Sobolev extension domains. Assume that $\Omega \subset$ $\mathbb{R}^{n}$ is a bounded domain and its boundary $\partial \Omega$ is $m$-regular, $n-1 \leq m<n$. Then the following two conditions are equivalent for every $p, n-1<p<\infty$ :

(1) The domain $\Omega$ is a $W^{1, p}$-extension domain.

(2) There exists a bounded linear trace operator

$$
T: W^{1, p}(\Omega) \rightarrow B_{1-\frac{n-m}{p}}^{p}(\partial \Omega)
$$

so that $T u(x)=\lim _{r \rightarrow 0} f_{B^{n}(x, r) \cap \Omega} u(z) d H^{n}(z) H^{m}$-almost everywhere in $\partial \Omega$ and Lipschitz continuous functions are dense in $W^{1, p}(\Omega)$.

\section{NotATION AND PRELIMINARIES}

Throughout this paper $C$ will denote a constant which may change even in a single string of an estimate. We let $\Omega$ be a domain of Euclidean n-space $\mathbb{R}^{n}, n \geq 2$. The norm of $x \in \mathbb{R}^{n}$ is $|x|=\sqrt{x_{1}^{n}+\ldots+x_{n}^{2}}$. By an open ball centered at $x$ and with radius $r>0$ we mean the set $B^{n}(x, r)=\left\{y \in \mathbb{R}^{n}:|y-x|<r\right\}$. By an open cube we mean the set $Q(x, r)=\left\{y \in \mathbb{R}^{n}: \max _{j=1, . ., n}\left|y_{j}-x_{j}\right|<r\right\}$, and we write $k Q$ for the cube with the same center as $Q$ and dilated by a factor $k>0$. We let $\operatorname{diam}(A)=\sup \{|x-y|: x, y \in A\}$ denote the diameter of a set $A$. The boundary of a domain $\Omega$ is denoted by $\partial \Omega$. Let $|A|$ be the Lebesgue $n$-measure of a set $A$. Let $H^{m}$ be the $m$-dimensional Hausdorff measure. We write $u \approx v$ if there exists a constant $C \geq 1$ so that $C^{-1} u \leq v \leq C u$. By $f_{A} u(x) d x$ we denote the integral average of the function $u$ over the set $A$ with $|A|>0$.

We say that a bounded set $F \subset \mathbb{R}^{n}$ is $m$-regular, $0<m \leq n$, if there exists a constant $C>0$ so that

$$
C^{-1} r^{m} \leq H^{m}\left(B^{n}(x, r) \cap F\right) \leq C r^{m}
$$

for every $x \in F$ and every $r, 0<r \leq \operatorname{diam}(F)$. A typical example of an $m$-regular set is the boundary of Koch's snowflake domain with $m=\frac{\ln 4}{\ln 3}$. For more details about m-regular sets we refer to [6, Chapter II].

By $C(A)$ we denote the set of continuous functions in a set $A$. By $C^{0, \lambda}(A)$, $0<\lambda \leq 1$, we denote the set of $\lambda$-Hölder continuous functions $u \in C^{0, \lambda}(A)$ if there exists a constant $C>0$ so that

$$
|u(x)-u(y)| \leq C|x-y|^{\lambda}
$$

for every $x, y \in A$. If $\lambda=1$ we say that the function $u$ is Lipschitz continuous.

By $L^{p}(D), 1 \leq p \leq \infty$, we denote the class of all $p$-integrable functions in an open set $D \subset \mathbb{R}^{n}$. By $L^{1, p}(D), 1 \leq p \leq \infty$, we denote the class of all functions whose first weak partial derivatives belong to $L^{p}(D)$. The classical Sobolev space in an open set $D$ is denoted by $W^{1, p}(D)=L^{p}(D) \cap L^{1, p}(D), 1 \leq p \leq \infty$. We equip $W^{1, p}(D)$ with the norm $\|u\|_{W^{1, p}(D)}=\|u\|_{L^{p}(D)}+\|\nabla u\|_{L^{p}(D)}$, where $\nabla u$ is the weak gradient. 
By $M^{1, p}(D), 1<p \leq \infty$, we denote the Hajłasz space $u \in M^{1, p}(D)$ if $u \in L^{p}(D)$, and there exists a non-negative $g \in L^{p}(D)$ so that

$$
|u(x)-u(y)| \leq|x-y|(g(x)+g(y))
$$

for almost every $x, y \in D$. For an arbitrary domain $\Omega$ it holds that $M^{1, p}(\Omega) \subset$ $W^{1, p}(\Omega)$. If a domain $\Omega$ is a $W^{1, p}$-extension domain, then $M^{1, p}(\Omega)=W^{1, p}(\Omega)$, [1, Theorem 1, p. 405]. These spaces were defined by P. Hajłasz, [1].

Let $F \subset \mathbb{R}^{n}$ be an $m$-regular set, $0<m \leq n$. The Besov space $B_{1-\frac{n-m}{p}}^{p}(F)$, $1<p<\infty$, consists of all functions $u \in L^{p}(F)$ for which

$$
\iint_{|x-y|<1} \frac{|u(x)-u(y)|^{p}}{|x-y|^{p-n+2 m}} d H^{m}(x) d H^{m}(y)<\infty .
$$

The Besov space is equipped with the norm

$$
\|u\|_{B_{1-\frac{n-m}{p}}^{p}}(F)=\|u\|_{L^{p}(F)}+\left(\iint_{|x-y|<1} \frac{|u(x)-u(y)|^{p}}{|x-y|^{p-n+2 m}} d H^{m}(x) d H^{m}(y)\right)^{\frac{1}{p}} .
$$

More details about classical Besov spaces and Besov spaces on an $m$-regular set can be found for example in [8, Sections 8.3 and 8.4] and [6, Chapter V].

We say that a domain $\Omega \subset \mathbb{R}^{n}$ is a $W^{1, p}$-extension domain if there exists a bounded linear extension operator

$$
E: W^{1, p}(\Omega) \rightarrow W^{1, p}\left(\mathbb{R}^{n}\right),
$$

such that $\left.E u\right|_{\Omega}=u$ and a constant $C>0$, so that

$$
\|E u\|_{W^{1, p}\left(\mathbb{R}^{n}\right)} \leq C\|u\|_{W^{1, p}(\Omega)}
$$

for every $u \in W^{1, p}(\Omega)$.

\section{EXTENSION OPERATOR}

First we construct an extension operator. Let $\Omega \subset \mathbb{R}^{n}$ be a bounded domain and let its boundary $\partial \Omega$ be $m$-regular, $n-1 \leq m<n$. In this chapter we assume there exists a bounded trace operator $T: W^{1, p}(\Omega) \rightarrow B_{1-\frac{n-m}{p}}^{p}(\partial \Omega), 1<p<\infty$, such that

$$
T u(x)=\lim _{r \rightarrow 0} f_{B^{n}(x, r) \cap \Omega} u(z) d H^{n}(z)
$$

$H^{m}$-almost everywhere in $\partial \Omega$. Let $\mathcal{W}_{0}$ be the Whitney decomposition of $\mathbb{R}^{n} \backslash \bar{\Omega}$, [10, Theorem 1, p. 167]. Let $\mathcal{W}$ be the family of those cubes $Q_{i} \in \mathcal{W}_{0}$, for which $\operatorname{diam}\left(Q_{i}\right)<\min \{a, \operatorname{diam}(\Omega)\}$ for every $i$. Here $0<a \leq 1$ is a constant which we will fix in the proof of Lemma 3.4. We will write that $W=\bigcup_{Q \in \mathcal{W}} Q$. Let $\left(\phi_{i}\right)$ be a partition of unity corresponding to the collection $\mathcal{W}_{0}: 0 \leq \phi_{i} \leq 1, \phi_{i} \in C_{0}^{\infty}\left(\frac{9}{8} Q_{i}\right)$, $\left|\nabla \phi_{i}\right| \leq C \operatorname{diam}\left(Q_{i}\right)^{-1}$ and $\sum_{i} \phi_{i}(x)=1$ for every $x \in \mathbb{R}^{n} \backslash \bar{\Omega}$; see [10, p. 170]. For each cube $Q_{i} \in \mathcal{W}$ we pick $x_{i} \in \partial \Omega$ so that $\operatorname{dist}\left(Q_{i}, \partial \Omega\right)=\operatorname{dist}\left(Q_{i}, x_{i}\right)$. Let

$$
a_{i}=f_{B^{n}\left(x_{i}, r_{i}\right) \cap \partial \Omega} T u(y) d H^{m}(y),
$$

where $T u \in B_{1-\frac{n-m}{p}}^{p}(\partial \Omega)$ and $r_{i}=\operatorname{diam}\left(Q_{i}\right)$. If $Q_{i} \notin \mathcal{W}$ we set $a_{i}=0$. 
We define an extension operator $E: W^{1, p}(\Omega) \rightarrow W^{1, p}\left(\mathbb{R}^{n}\right)$ by setting

$$
E u(x)=\left\{\begin{array}{rll}
u(x), & \text { for } & x \in \Omega \\
\sum_{i=1}^{\infty} a_{i} \phi_{i}(x), & \text { for } & x \in \mathbb{R}^{n} \backslash \bar{\Omega} .
\end{array}\right.
$$

Note that the extension operator is the same for every $p$. It is also easy to see that $E u \in C^{\infty}\left(\mathbb{R}^{n} \backslash \bar{\Omega}\right)$ and $E u$ is zero in the complement of $\bigcup_{Q_{i} \in \mathcal{W}} \frac{9}{8} Q_{i} \cup \bar{\Omega}$. The extension operator is equivalent to counting the sum only over those $\phi_{i}$ with $Q_{i} \in \mathcal{W}$.

For technical reasons we have chosen a slightly different extension operator than Jonsson [4, Theorem 3, p. 4]. For every cube we first pick the nearest point $x_{i}$ from the boundary and then set the value of $a_{i}$; see (3.1). Jonsson just sets

$$
a_{i}^{*}=f_{B^{n}\left(z_{i}, C r_{i}\right) \cap \partial \Omega} T u(y) d H^{m}(y),
$$

where $z_{i}$ is the center of the cube $Q_{i} \in \mathcal{W}_{0}, r_{i}=\operatorname{diam}\left(Q_{i}\right)$ and $C>0$ is a constant.

3.2. Lemma. Let $1<p<\infty$. Assume that $\Omega \subset \mathbb{R}^{n}$ is a bounded domain and its boundary $\partial \Omega$ is $m$-regular, $n-1 \leq m<n$. If there exists a bounded trace operator $T: W^{1, p}(\Omega) \rightarrow L^{p}(\partial \Omega)$, then there exists a constant $C, 0<C<\infty$, such that

$$
\|E u\|_{L^{p}\left(\mathbb{R}^{n}\right)} \leq C\|u\|_{W^{1, p}(\Omega)}
$$

for every $u \in W^{1, p}(\Omega)$.

Proof. Let $x \in \frac{9}{8} Q_{0}$ for some $Q_{0} \in \mathcal{W}$. Hence we obtain

$$
|E u(x)|=\left|\sum_{i=1}^{\infty} a_{i} \phi_{i}(x)\right| \leq C \sup _{i}\left|a_{i}\right|
$$

where the supremum is taken over all those cubes $Q_{i} \in \mathcal{W}$ for which $\frac{9}{8} Q_{i} \cap \frac{9}{8} Q_{0} \neq \emptyset$. Since $\partial \Omega$ is $m$-regular and $r_{i}=\operatorname{diam}\left(Q_{i}\right)$, we obtain

$$
\begin{aligned}
\sup _{i}\left|a_{i}\right| & \leq \sup _{i}\left(f_{B^{n}\left(x_{i}, r_{i}\right) \cap \partial \Omega}|T u|^{p} d H^{m}\right)^{\frac{1}{p}} \\
& \leq C \operatorname{diam}\left(Q_{0}\right)^{-\frac{m}{p}}\left(\int_{B^{n}\left(x_{0}, C r_{0}\right) \cap \partial \Omega}|T u|^{p} d H^{m}\right)^{\frac{1}{p}} .
\end{aligned}
$$

Here $C>0$ is a constant so that $B^{n}\left(x_{i}, r_{i}\right) \subset B^{n}\left(x_{0}, C r_{0}\right)$ for every $i, \frac{9}{8} Q_{i} \cap \frac{9}{8} Q_{0} \neq$ $\emptyset$. It is possible to choose this constant, because for every two touching cubes the distance of corresponding points in boundary is comparable to the diameters of cubes. Note that $\mathcal{W}$ is a collection of Whitney cubes with $\operatorname{diam}\left(Q_{i}\right)<$ $\min \{a, \operatorname{diam}(\Omega)\}$ for every $i=1,2, \ldots$ Let $N_{k}$ be a number of cubes from the $\mathrm{k}^{\text {th }}$ generation in the Whitney decomposition; then $r_{j} \approx 2^{-k}$. We write $Q_{i}^{k}$, 
$i=1, \ldots, N_{k}$, to denote the Whitney cubes from the $\mathrm{k}^{\text {th }}$ generation. There exists an integer $k_{0}$ such that $Q_{i}^{k} \in \mathcal{W}$ if and only if $k \geq k_{0}$. This implies

$$
\begin{aligned}
\int_{R^{n} \backslash \bar{\Omega}}|E u|^{p} d H^{n} & \leq \sum_{k=k_{0}}^{\infty} \sum_{i=1}^{N_{k}} \int_{\frac{9}{8} Q_{i}^{k}}|E u|^{p} d H^{n} \\
& \leq \sum_{k=k_{0}}^{\infty} \sum_{i=1}^{N_{k}} C 2^{-k(-m)} \int_{B^{n}\left(x_{i}, C r_{i}\right) \cap \partial \Omega}|T u|^{p} d H^{m} \cdot\left|Q_{i}^{k}\right| \\
& \leq \sum_{k=k_{0}}^{\infty} \sum_{i=1}^{N_{k}} C 2^{-k(n-m)} \int_{B^{n}\left(x_{i}, C r_{i}\right) \cap \partial \Omega}|T u|^{p} d H^{m} \\
& \leq C \sum_{k=k_{0}}^{\infty} 2^{-k(n-m)} \int_{\partial \Omega}|T u|^{p} d H^{m} \\
& \leq C\|T u\|_{L^{p}(\partial \Omega)}^{p} .
\end{aligned}
$$

The second-to-last inequality follows from the fact that no point belongs to more than $C(n)$ balls $B^{n}\left(x_{i}, C r_{i}\right), i=1, \ldots, N_{k}$. To see that, let $x \in \partial \Omega$. Assume that the point $x$ belongs to the balls $B^{n}\left(x_{i}, C r_{i}\right), i=1,2,3, \ldots$. Since $\operatorname{dist}\left(Q_{i}, x\right) \leq$ $C 2^{-k}$ and $\left|Q_{i}\right|=2^{-k n}$ for every $i=1,2,3, \ldots$, and interiors of Whitney cubes are disjoint, we obtain that the point $x$ cannot belong to more than $\left(\frac{C 2^{-k}}{2^{-k}}\right)^{n}=C^{n}$ balls $B^{n}\left(x_{i}, C r_{i}\right)$. There exists a bounded trace operator, and hence we obtain

$$
\|E u\|_{L^{p}\left(\mathbb{R}^{n}\right)} \leq C\|u\|_{W^{1, p}(\Omega)} .
$$

This completes the proof of Lemma 3.2

3.4. Lemma. Let $1<p<\infty$. Assume that $\Omega \subset \mathbb{R}^{n}$ is a bounded domain and its boundary $\partial \Omega$ is m-regular, $n-1 \leq m<n$. If there exists a bounded trace operator $T: W^{1, p}(\Omega) \rightarrow B_{1-\frac{n-m}{p}}^{p}(\partial \Omega)$, then there exists a constant $C, 0<C<\infty$, such that

$$
\|\nabla E u\|_{L^{p}\left(\mathbb{R}^{n} \backslash \partial \Omega\right)} \leq C\|u\|_{W^{1, p}(\Omega)}
$$

for every $u \in W^{1, p}(\Omega)$.

Proof. If $Q_{i}$ and $Q_{0}$ are two Whitney cubes from $\mathcal{W}$, with $\frac{9}{8} Q_{i} \cap \frac{9}{8} Q_{0} \neq \emptyset$, then $\operatorname{diam}\left(Q_{0}\right) \approx \operatorname{diam}\left(Q_{i}\right)$ and furthermore $\left|x_{i}-x_{0}\right| \leq C \operatorname{diam}\left(Q_{0}\right)$, where $\operatorname{dist}\left(Q_{0}, \partial \Omega\right)$ $=\operatorname{dist}\left(Q_{0}, x_{0}\right), x_{0} \in \partial \Omega$, and $\operatorname{dist}\left(Q_{i}, \partial \Omega\right)=\operatorname{dist}\left(Q_{i}, x_{i}\right), x_{i} \in \partial \Omega$. We obtain for these cubes $Q_{i}$ and $Q_{0}$ that

$$
\begin{aligned}
& \left|a_{i}-a_{0}\right|=\left|\underset{B^{n}\left(x_{i}, r_{i}\right) \cap \partial \Omega}{f} T u(z) d H^{m}(z)-\underset{B^{n}\left(x_{0}, r_{0}\right) \cap \partial \Omega}{f} T u(w) d H^{m}(w)\right| \\
& \quad \leq\left(\underset{B^{n}\left(x_{i}, r_{i}\right) \cap \partial \Omega}{f} f_{B^{n}\left(x_{0}, r_{0}\right) \cap \partial \Omega}|T u(z)-T u(w)|^{p} d H^{m}(z) d H^{m}(w)\right)^{\frac{1}{p}} \\
& \quad \leq C\left(r_{0}^{-2 m}\right. \\
& \left.\quad \cdot \int_{B^{n}\left(x_{0}, C r_{0}\right) \cap \partial \Omega} \int_{B^{n}\left(x_{0}, r_{0}\right) \cap \partial \Omega}|T u(z)-T u(w)|^{p} d H^{m}(z) d H^{m}(w)\right)^{\frac{1}{p}} .
\end{aligned}
$$


The constant $C$ does not depend on the cube $Q_{0}$. Assume that $Q_{j} \in \mathcal{W}$ is a Whitney cube from the $\mathrm{k}^{\text {th }}$ generation; then $r_{j} \approx 2^{-k}$. Let $x \in Q_{j}$. This implies that

$$
|\nabla E u(x)|=\left|\nabla \sum_{i=1}^{\infty} a_{i} \phi_{i}(x)\right|=\left|\sum_{i=1}^{\infty}\left(a_{i}-a_{j}\right) \nabla \phi_{i}(x)\right|
$$

since $\sum_{i} \phi_{i}(x)=1$ for every $x \in \mathbb{R}^{n} \backslash \bar{\Omega}$. We obtain

$$
\begin{aligned}
& \int_{Q_{j}}|\nabla E u|^{p} d H^{n} \leq C \sup _{Q_{i} \cap Q_{j} \neq \emptyset}\left|a_{i}-a_{j}\right|^{p} C \operatorname{diam}\left(Q_{j}\right)^{-p}\left|Q_{j}\right| \\
& \leq C 2^{2 k m} 2^{k p} 2^{-k n} \\
& \cdot \int_{B^{n}\left(x_{j}, C r_{j}\right) \cap \partial \Omega} \int_{B^{n}\left(x_{j}, r_{j}\right) \cap \partial \Omega}|T u(z)-T u(w)|^{p} d H^{m}(w) d H^{m}(z) \\
& \leq C 2^{-k(n-p-2 m)} \\
& \cdot \int_{B^{n}\left(x_{j}, C r_{j}\right) \cap \partial \Omega} \int_{B^{n}\left(x_{j}, r_{j}\right) \cap \partial \Omega}|T u(z)-T u(w)|^{p} d H^{m}(w) d H^{m}(z) .
\end{aligned}
$$

We write $W=\bigcup_{Q \in \mathcal{W}} Q$. This implies

$$
\begin{aligned}
& \int_{W}|\nabla E u|^{p} d H^{m} \leq \sum_{k=k_{0}}^{\infty} \sum_{j=1}^{N_{k}} \int_{Q_{j}}|\nabla E u|^{p} d H^{n} \\
& \leq C \sum_{k=k_{0}}^{\infty} \sum_{j=1}^{N_{k}} 2^{-k(n-p-2 m)} \\
& \cdot \int_{B^{n}\left(x_{j}, C r_{j}\right) \cap \partial \Omega} \int_{B^{n}\left(x_{j}, r_{j}\right) \cap \partial \Omega}|T u(z)-T u(w)|^{p} d H^{m}(w) d H^{m}(z) .
\end{aligned}
$$

Every point $x \in \partial \Omega$ belongs only to a uniformly bounded number of balls $B^{n}\left(x_{j}, r_{j}\right)$ from the $\mathrm{k}^{\text {th }}$ generation in the Whitney decomposition. This yields

$$
\begin{aligned}
& \int_{W}|\nabla E u|^{p} d H^{n} \leq C \sum_{k=k_{0}}^{\infty} 2^{-k(n-p-2 m)} \\
& \cdot \iint_{|z-w|<C 2^{-k}}|T u(z)-T u(w)|^{p} d H^{m}(w) d H^{m}(z) \\
& =C \sum_{k=k_{0}}^{\infty} \iint_{|z-w|<C 2^{-k}} \frac{|T u(z)-T u(w)|^{p}}{2^{-k(p-n+2 m)}} d H^{m}(w) d H^{m}(z) .
\end{aligned}
$$

Note that $\mathcal{W}$ is a collection of Whitney cubes $Q \in \mathcal{W}_{0}$ with

$$
\operatorname{diam}(Q) \leq \min \{a, \operatorname{diam}(\Omega)\} .
$$

We choose that $a$ is small enough to guarantee that $C 2^{-k_{0}}<1$. By [5, Lemma 5.3, p. 166] we obtain

$$
\int_{W}|\nabla E u|^{p} d H^{n} \leq C \iint_{|z-w|<1} \frac{|T u(z)-T u(w)|^{p}}{|z-w|^{p-n+2 m}} d H^{m}(w) d H^{m}(z) .
$$


Let $Q_{1}^{k_{0}}, \ldots, Q_{N}^{k_{0}}$ be the largest generation of the Whitney cubes belonging to $\mathcal{W}$. Note that $\operatorname{diam}\left(Q_{i}^{k_{0}}\right)$ depends on $\min \{a, \operatorname{diam}(\Omega)\}$. We write $A=\left(\bigcup_{i=1}^{N} \frac{9}{8} Q_{i}^{k_{0}}\right) \backslash$ $W$. Assume that $x \in A$ and $x \in \frac{9}{8} Q_{i}^{k_{0}}$. This yields

$$
\begin{aligned}
|\nabla E u(x)| & =\left|\nabla \sum_{i=1}^{N} a_{i} \phi_{i}(x)\right| \\
& \leq C \sup _{j}\left|a_{j}\right| \cdot \frac{C}{\operatorname{diam}\left(Q_{i}^{k_{0}}\right)} \\
& \leq C \sup _{j}\left|a_{j}\right| .
\end{aligned}
$$

Here the supremum is taken over all cubes $\frac{9}{8} Q_{j}^{k_{0}} \cap \frac{9}{8} Q_{i}^{k_{0}} \neq \emptyset$. As in (3.3) we obtain

$$
\sup _{i}\left|a_{i}\right| \leq C\left(\int_{B^{n}\left(x_{i}, C r_{0}\right) \cap \partial \Omega}|T u|^{p} d H^{m}\right)^{\frac{1}{p}}
$$

here $r_{0}=\operatorname{diam}\left(Q_{i}^{k_{0}}\right)$. This implies

$$
\int_{A}|\nabla E u(x)|^{p} d H^{n} \leq \sum_{i=1}^{N} C \int_{B^{n}\left(x_{i}, C r_{0}\right) \cap \partial \Omega}|T u|^{p} d H^{m} .
$$

Since no point in $\partial \Omega$ belongs to more than $C(n)$ balls $B^{n}\left(x_{i}, C r_{0}\right)$ we obtain

$$
\int_{A}|\nabla E u(x)|^{p} d H^{n}(x) \leq C\|T u\|_{L^{p}(\partial \Omega)}^{p} .
$$

In $\mathbb{R}^{n} \backslash(\bar{\Omega} \cup W \cup A)$ the function $E u$ is zero. Since there exists a bounded trace operator we obtain by (3.5) and (3.6) that

$$
\|\nabla E u\|_{L^{p}\left(\mathbb{R}^{n} \backslash \partial \Omega\right)} \leq C\|u\|_{W^{1, p}(\Omega)} .
$$

This completes the proof of Lemma 3.4

The extension of $u \in W^{1, p}(\Omega)$ is a $C^{\infty}$-function in $\mathbb{R}^{n} \backslash \bar{\Omega}$. We are left to prove that the weak gradient of $E u$ exists across the boundary. Lemma 3.7 follows easily from the construction of the extension operator.

3.7. Lemma. Let $1<p<\infty$ and $0<\lambda \leq 1$. Assume that $\Omega \subset \mathbb{R}^{n}$ is a bounded domain and there exists a trace operator $T$ to the boundary $\partial \Omega$ so that $T u=\left.u\right|_{\partial \Omega}$ for every $u \in W^{1, p}(\Omega) \cap C(\bar{\Omega})$. If $u \in W^{1, p}(\Omega) \cap C^{0, \lambda}(\Omega)$, then $E u \in C^{0, \lambda}\left(\mathbb{R}^{n}\right)$.

The following result is due to Hajłasz and Martio, [2, Lemma 11, p. 237]: If a compact set $K \subset \mathbb{R}^{n}$ satisfies $H^{n-\lambda}(K)=0$, where $0 \leq \lambda<1$, then for every p, $1 \leq p \leq \infty$,

$$
W^{1, p}\left(\mathbb{R}^{n} \backslash K\right) \cap C^{0,1-\lambda}\left(\mathbb{R}^{n}\right) \subset W^{1, p}\left(\mathbb{R}^{n}\right) .
$$

Since $\partial \Omega$ is $m$-regular, $H^{n-\lambda}(\partial \Omega)=0$ for every $\lambda, 0 \leq \lambda<n-m$, the general case follows by Lemmata $3.2,3.4$ and 3.7 when we assume that $W^{1, p}(\Omega) \cap C^{0,1-\lambda}(\Omega)$ is dense in $W^{1, p}(\Omega)$ for some $\lambda, 0 \leq \lambda<n-m$.

3.8. Theorem. Let $1<p<\infty$. Let $\Omega \subset \mathbb{R}^{n}$ be a bounded domain so that its boundary $\partial \Omega$ is $m$-regular, $n-1 \leq m<n$. Assume that $W^{1, p}(\Omega) \cap C^{0,1-\lambda}(\Omega)$ is dense in $W^{1, p}(\Omega)$ for some $\lambda, 0 \leq \lambda<n-m$. If there exists a bounded linear trace operator

$$
T: W^{1, p}(\Omega) \rightarrow B_{1-\frac{n-m}{p}}^{p}(\partial \Omega),
$$


so that $T u=\left.u\right|_{\partial \Omega}$ for every $u \in W^{1, p}(\Omega) \cap C(\bar{\Omega})$, then the domain $\Omega$ is a $W^{1, p}$ extension domain: there exists a bounded linear extension operator

$$
E: W^{1, p}(\Omega) \rightarrow W^{1, p}\left(\mathbb{R}^{n}\right),
$$

so that $\left.E u\right|_{\Omega}=u$ for every $u \in W^{1, p}(\Omega)$.

3.9. Remark. If $\Omega \subset \mathbb{R}^{n}$ is a bounded $W^{1, p}$-extension domain, $p>n-1$, then $H^{n}(\partial \Omega)=0$, [7, Theorem 6.5, p. 28] and [3, Corollary 4.9, p. 184]. Thus it is natural to assume that $m$ is strictly less than $n$. Lipschitz continuous functions are dense in $W^{1, p}(\Omega)$ if $M^{1, p}(\Omega)=W^{1, p}(\Omega)$, [1, Theorem 5, p. 408]. In particular $M^{1, p}(\Omega)=W^{1, p}(\Omega)$ if $\Omega$ is a $W^{1, p}$-extension domain, 1, Theorem 1, p. 405]. The reverse does not hold; there exists a bounded domain $\Omega \subset \mathbb{R}^{n}$ with $M^{1, p}(\Omega)=W^{1, p}(\Omega)$, but $\Omega$ is not a $W^{1, p}$-extension domain, [9]. It is an open problem whether the condition about the density of Hölder continuous functions is necessary in Theorem 3.8

By the proof of Lemmata 3.2 and 3.4 we obtain the following corollary.

3.10. Corollary. Let $1<p<\infty$. Assume that $\Omega \subset \mathbb{R}^{n}$ is a bounded domain and its boundary $\partial \Omega$ is $m$-regular, $n-1 \leq m<n$. If there exists a bounded trace operator $T: W^{1, p}(\Omega) \rightarrow B_{1-\frac{n-m}{p}}^{p}(\partial \Omega)$, then there exists a bounded linear extension operator

$$
E^{*}: B_{1-\frac{n-m}{p}}^{p}(\partial \Omega) \rightarrow W^{1, p}(\Omega)
$$

\section{Characterization for Sobolev extension domains}

Using Theorem 3.8 we give a characterization for Sobolev extension domains. We need the following trace theorem, which is due to Jonsson.

4.1. Theorem (44, Theorem 3, p. 4], 6, Theorem 1, p. 182]). Let $1<p<\infty$. Assume that $\Omega \subset \mathbb{R}^{n}$ is a bounded domain and its boundary $\partial \Omega$ is m-regular, $n-1 \leq m<n$. Then the operator

$$
T: W^{1, p}\left(\mathbb{R}^{n}\right) \rightarrow B_{1-\frac{n-m}{p}}^{p}(\partial \Omega),
$$

$T u(x)=\lim _{r \rightarrow 0} f_{B^{n}(x, r)} u(z) d H^{n}(z) H^{m}$-almost everywhere in $\partial \Omega$, is bounded and linear.

Assume that $\Omega \subset \mathbb{R}^{n}$ is a bounded domain and its boundary $\partial \Omega$ is $m$-regular, $n-1 \leq m<n$. We have to show that if $\Omega$ is a $W^{1, p}$-extension domain, then

$$
\lim _{r \rightarrow 0} f_{B^{n}(x, r) \cap \Omega} u(z) d H^{n}(z)=\lim _{r \rightarrow 0} f_{B^{n}(x, r)} E u(z) d H^{n}(z),
$$

for every $x \in \partial \Omega \backslash F$, with $H^{m}(F)=0$.

The proof is similar to the proof in [11, Theorem 1, p. 121] as soon as we know that $\Omega$ is $n$-regular. The case $m=n-1$ has been proved in [6, Proposition 2, p. 206]. A bounded $W^{1, p}$-extension domain, $n-1<p<\infty$, is $n$-regular by [7, Theorem 6.5, p. 28] and [3, Corollary 4.9, p. 184]. 
By Theorem 4.1 we obtain the following result.

4.2. Corollary. Let $n-1<p<\infty$. Assume that $\Omega \subset \mathbb{R}^{n}$ is a bounded $W^{1, p}$ extension domain and its boundary $\partial \Omega$ is $m$-regular, $n-1 \leq m<n$. Then there exists a bounded linear trace operator

$$
T: W^{1, p}(\Omega) \rightarrow B_{1-\frac{n-m}{p}}^{p}(\partial \Omega),
$$

so that $T u(x)=\lim _{r \rightarrow 0} f_{B^{n}(x, r) \cap \Omega} u(z) d H^{n}(z) H^{m}$-almost everywhere in $\partial \Omega$.

By Theorem 3.8, Corollary 4.2 and Remark 3.9 we obtain the following characterization for Sobolev extension domain.

4.3. Theorem. Let $n-1<p<\infty$. Assume that $\Omega \subset \mathbb{R}^{n}$ is a bounded domain and its boundary $\partial \Omega$ is $m$-regular, $n-1 \leq m<n$. Then the following two conditions are equivalent:

(1) The domain $\Omega$ is a $W^{1, p}$-extension domain: there exists a bounded linear extension operator

$$
E: W^{1, p}(\Omega) \rightarrow W^{1, p}\left(\mathbb{R}^{n}\right),
$$

such that $\left.E u\right|_{\Omega}=u$ for every $u \in W^{1, p}(\Omega)$.

(2) There exists a bounded linear trace operator

$$
T: W^{1, p}(\Omega) \rightarrow B_{1-\frac{n-m}{p}}^{p}(\partial \Omega),
$$

such that $T u(x)=\lim _{r \rightarrow 0} f_{B^{n}(x, r) \cap \Omega} u(z) d H^{n}(z) H^{m}$-almost everywhere in $\partial \Omega$, and Lipschitz continuous functions are dense in $W^{1, p}(\Omega)$.

4.4. Remark. By Theorem 3.8 the set of Lipschitz continuous functions in Theorem 4.3 (2) can be replaced by the set $W^{1, p}(\Omega) \cap C^{0,1-\lambda}(\Omega)$, where $0 \leq \lambda<n-m$.

\section{ACKNOWLEDGMENTS}

I thank P. Hajłasz for suggesting that I study this topic, my teacher R. HurriSyrjänen for her helpful guidance and the referee for many crucial comments and corrections.

\section{REFERENCES}

[1] P. Hajłasz: Sobolev spaces on an arbitrary metric space, Potential Anal. 5(4) (1996), 403415. MR1401074 (97f:46050)

[2] P. Hajłasz and O. Martio: Traces of Sobolev functions on fractal type sets and characterization of extension domains, J. Funct. Anal. 143(1) (1997), 221-246. MR1428124 (98d:46034)

[3] D. A. Herron and P. Koskela: Uniform, Sobolev extension and quasiconformal circle domains, J. Anal. Math. 57 (1991), 172-202. MR1191746 (94m:46059)

[4] A. Jonsson: The trace of potentials on general sets, Ark. Mat. 17(1) (1979), 1-18. MR0543499 (80i:46029)

[5] A. Jonsson and H. Wallin: A Whitney extension theorem in $L_{p}$ and Besov spaces, Ann. Inst. Fourier, Grenoble 28(1) (1978), 139-192. MR0500920 (81c:46024)

[6] A. Jonsson and H. Wallin: Function spaces on subsets of $\mathbb{R}^{n}$, Harwood Academic Publisher, London, 1984, Mathematical Reports, Volume 2, Part 1. MR0820626 (87f:46056)

[7] P. Koskela: Capacity extension domains, Ann. Acad. Sci. Fenn. Ser. A I Math. Dissertationes 73 (1990), 1-42. MR 1039115 (91e:46041)

[8] A. Kufner, O. John and S. Fučík: Function spaces, Noordhoff International Publishing, Leyden, 1977. MR0482102 (58:2189) 
[9] A. S. Romanov: On a generalization of Sobolev spaces, Siberian Math. J. 39(4) (1998), 821-824. MR1654102 (99k:46059)

[10] E. M. Stein: Singular integrals and differentiability properties of functions, Princeton University Press, Princeton, N.J., 1970. MR0290095 (44:7280)

[11] H. Wallin: The trace to the boundary of Sobolev spaces on a snowflake, Manuscripta Math. 73(2) (1991), 117-125. MR.1128682 (92k:46053)

Department of Mathematics and Statistics, P.O. Box 68 (Gustav Hällströmin katu 2B), Fin-00014 University of Helsinki, Finland

E-mail address: petteri.harjulehto@helsinki.fi 